\title{
Pierre Meulien
}

The Innovative Medicines Initiative (IMI) is the largest pharmaceuticalfocused public-private partnership (PPP) in the world. Last year, funders committed a further $€ 3.3$ billion to the 7-year-old initiative, which already spent $€ 2$ billion in its first phase of operations. The IMI now has 50 projects in its pipeline, spanning neurological conditions, oncology, antimicrobials and more. Starting in September, it will also have a new executive director, with Pierre Meulien stepping into the role. Meulien has decades of experience in both the private and the public sector - including time spent as Senior Vice President of Research and Development at Aventis Pasteur and, most recently, as President of the non-profit Genome Canada, which supports large-scale genomics and proteomics research in Canada. He tells Asher Mullard about his plans for the IMI.

\section{Q What drew you to the IMI position?}

I've always been fascinated by the interface between the public and private sectors. When I worked in the private sector, I felt that we weren't taking advantage of a lot of stuff that was going on in the public sector. The IMI is right in the sweet spot between these sectors. And it is really of a scale that has potential to have impact, so that's what excites me about this venture. It is the largest PPP in the world in this space, and I think it really is the way to go.

\section{Q What's your vision for the IMI?}

The IMI already has a good strategy in terms of which topics it works on. They've chosen some no-brainers: of course we want to use existing drugs more efficiently, and to bring new drugs where there is medical need. The question now is really how we can implement some of the ideas and technologies the IMI has been working on. How do we take the reality of the genomics technologies, for example, and decide how to implement them in the clinic? Or how can you best change the clinical trial paradigm to make it more efficient?

I think that, with the maturity of the first round of IMI projects and the knowledge base we have established, we can now at least look at implementation. It's not that I have a magic bullet that I can deliver to answer these questions. It's really trying to be smarter about how we approach implementation. And I think that to do that, the next phase of the IMI is really going to have to get payers and regulatory people involved much more than they have been up to now.
Having said that, technology doesn't stop advancing. If you are going to set up a clinical genome-sequencing system in Europe, for example, at what point do you choose the platform that you are going to use, given that we know that in 5 years that platform is going to be very different to what it is today? Those are the kinds of things we have to deal with. Some people say it is like building an airplane while flying.

Q The IMI has come under fire recently from the German newspaper Der Spiegel and others, who have noted a lack of transparency from the IMI's pharmaceutical partners, and difficulties for small and medium-sized enterprises (SMEs) and academics to benefit from the IMI.

What do you make of these concerns? I think we need to take the criticisms seriously where they are valid and really look at what can be done.

In terms of the transparency, the IMI is going to take this very seriously, and I think members of the European Federation of Pharmaceutical Industries and Associations (EFPIA) are going to cooperate. I don't yet know the details about how diligence will

to get the most research impact

from all of the public research

dollars that are being spent

across the world, I think that,

at the very minimum, we have

to get together and inform each

other about what is going on

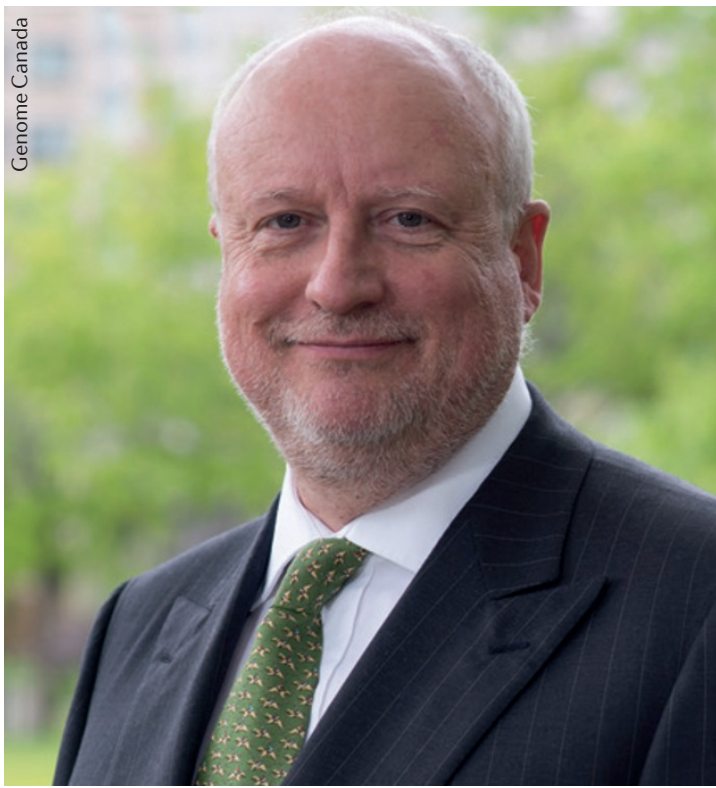

be performed. It's not that I want to increase the bureaucracy any more than is necessary. What interests me is getting funding to projects that are going to deliver something. But, having said that, I think that one of the reasons I was selected for this position is that I run an organization that has huge accountability for spending taxpayers' money, interacting with industry players and defining in-kind contributions.

We will also be looking at engagement with SMEs and academia very carefully. The importance of this engagement is huge.

\section{Q Although the IMI is the largest} biopharmaceutical PPP around, there is an explosion of other PPPs with overlapping biopharmaceutical interests. Is there a need to manage these overlaps, and for the IMI to start collaborating with other PPPs?

In order for us to get the most research impact from all of the public research dollars that are being spent across the world, I think that, at the very minimum, we have to get together and inform each other about what is going on. Just in terms of genomics, there is now the Precision Medicine Initiative, the US National Human Genome Research Institute's genomic medicine programmes and the 100,000 Genomes Project in the United Kingdom. The goal isn't necessarily to try to unify everybody into doing the same thing, though. We have to understand what everyone else is doing. We need to increase the amount of interaction between the IMI and others. And I think there is great value in some of these roadmaps - simple roadmaps — just to ensure that where there are different strategies in different countries, we are looking at these and taking learnings from one another. 